\title{
THE RADON IMAGE AS PLENOPTIC FUNCTION
}

\author{
Todor Georgiev Salil Tambe $^{\dagger} \quad$ Andrew Lumsdaine ${ }^{\star \dagger} \quad$ Jennifer Gille $^{\star} \quad$ Ashok Veeraraghavan ${ }^{\dagger}$ \\ * Qualcomm QTI, 3165 Kifer Road, Santa Clara, CA 95051 \\ $\dagger$ Rice University, 6100 Main MS-366, Houston, TX 77005 \\ ${ }^{\star \dagger}$ Computer Science Department, Indiana University, Bloomington, IN 47405
}

\begin{abstract}
We introduce a novel plenoptic function that can be directly captured or generated after the fact in plenoptic cameras. Whereas previous approaches represent the plenoptic function over a $4 \mathrm{D}$ ray space (as radiance or light field), we introduce the representation of the plenoptic function over a 3D plane space. Our approach uses the Radon plenoptic function instead of the traditional 4D plenoptic function to achieve $3 \mathrm{D}$ representation - which promises reduced size, making it suitable for use in mobile devices. Moreover, we show that the original 3D luminous density of the scene can be recovered via the inverse Radon transform. Finally, we demonstrate how various $3 \mathrm{D}$ views and differently-focused pictures can be rendered directly from this new representation.
\end{abstract}

\section{INTRODUCTION}

Images captured by traditional cameras suffer from certain limitations. For instance, the images do not record depth information, the plane of focus is fixed, and there is no way to change focus after capture. Plenoptic ("all-optic") cameras, which aim to record the complete four-dimensional $(4 D)$ radiance (or plenoptic function or light field), address these shortcomings but have their own design issues, such as properly trading-off spatial and angular resolution for capture and rendering [1-4]. Data captured by such cameras is four-dimensional even though the visible world is inherently threedimensionsal. One would expect that it should be possible to recover the $3 D$ densities in the scene from the captured $4 D$ radiance, and it is certainly desirable to do so for the purposes of data reduction and more natural representations $[5,6]$.

To address these issues, in this paper we propose a novel plenoptic function to represent the radiance with only three dimensions. We transform the plenoptic image defined over $4 D$ line space into a Radon image defined over $3 D$ plane space. Moreover, the original luminous density of the scene over $3 D$ point space is recovered by performing the inverse Radon transform. Our representation admits rendering of final images with the expected plenoptic effects such as different $3 D$ views and differently-focused pictures.

\section{THE RADON IMAGE}

\subsection{Radon Transform}

The Radon transform was introduced by Johann Radon in 1917 [9]. In $2 D$, the Radon transform converts a point function $f(x, y)$ into a line function $\widehat{f}_{\theta}(r)$, called the Radon image of $f$. It can be shown that if the function $f$ satisfies certain constraints, then it is possible to recover $f(x, y)$ from its Radon transform $\widehat{f}_{\theta}(r)$ [9-11]. The

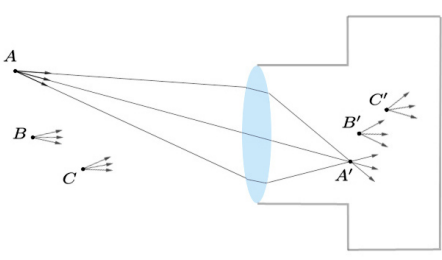

Fig. 1. Points in the real world are imaged as luminous points inside the camera where they can be detected by tomographic methods.

Radon transform is defined for any dimensionality. In $3 D$, the integral is computed over planes, while for $N$ dimensions, the transform maps the point function to a function defined over a set of $N-1$ dimensional hyperplanes $[9,12]$.

\subsection{Medical use of the Radon Transform}

In medical imaging, the Radon transform is used for non-invasive reconstruction of the internal structure of a body from integrals over large numbers of lines or planes passing through the body. The $3 D$ Radon image $\widehat{f}_{\phi, \theta}(r)$ is directly captured by passing radiation through the patient and measuring the summed densities while maintaining the appropriate geometry. In our notation, $r, \theta, \phi$ are polar coordinates of a point in $3 D$. The inverse Radon transform of the Radon image produces the $3 D$ density $f(x, y, z)$ inside the body.

\subsection{Radon Transform in Photography}

In computational photography, the $4 D$ radiance of a scene is known as the plenoptic function or the light field. Various mathematical representations have been considered since Gershun's 1936 paper [13] first sought to modernize the mathematical formalizations of light. The radiance describes the light energy as a function on the $4 D$ manifold of all rays in $3 D$. From this plenoptic function, an image is reconstructed that is focused at any depth chosen by the user, and as seen from an arbitrary point of view.

Our proposed novel use of the Radon transform is to represent the $4 D$ radiance by an alternative plenoptic function depending on only three variables instead of four. This function is the Radon image of the density of virtual light sources inside the camera (as shown in Fig. 1). (The Radon transform has previously been used in the context of plenoptic photography [7] as an alternative means of generating a focal stack rather than as alternative means of lightfield representation.) 
Any point $A$ in front of the camera is imaged into a luminous point $A^{\prime}$ behind the lens. A $3 D$ object is a large set of points $A, B, C, \ldots$, imaged into a large set of luminous points $A^{\prime}, B^{\prime}, C^{\prime}, \ldots$ behind the lens, creating a virtual $3 D$ object - a "point cloud" - inside the camera. Our end goal is effectively to capture the energy density of this virtual body as $f(x, y, z)$. The virtual body is cut with multiple thin virtual planes, the energy contained in each plane is measured, and the Radon image $\widehat{f}_{\phi, \theta}(r)$ constructed. This is analagous to approaches to computed tomography, only now applied to computational photography.

One advantage of our approach compared to prior art lightfield photography is that our plenoptic function is the Radon image, which is $3 D$ instead of $4 D$. This representation promises to be more economical in terms of memory requirements. This is a critical consideration because the large size of traditional $4 D$ plenoptic function files currently may not fit in the GPU memory of mobile devices.

Rendering an image from the Radon function $\widehat{f}_{\phi, \theta}(r)$ is accomplished by first performing the inverse Radon transform to generate the luminous density $f(x, y, z)$. This density is then projected onto an image plane that can be used to produce different views or different depths of focus. We describe our approach in more detail below.

\subsection{Integral Geometry}

Conceptually, our proposed approach has its place in the broader framework of integral geometry [12] (see Fig. 2).

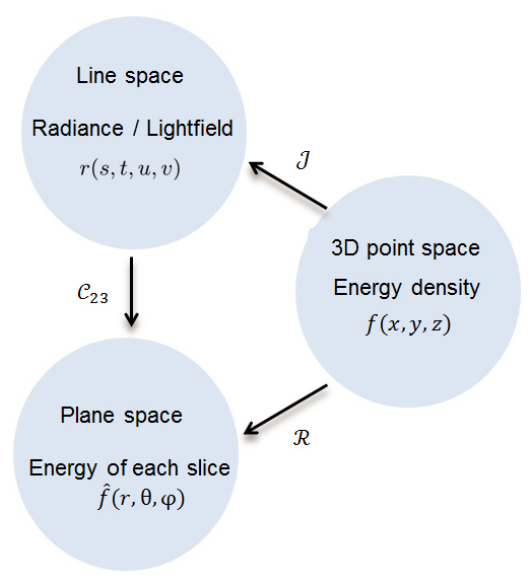

Fig. 2. Spaces and transforms in integral geometry that are directly applicable to light.

There are three representations of scene light energy, with corresponding mathematical spaces and transforms: (1) density in space; (2) radiance / lightfield; (3) energy as distributed over planes. The corresponding mathematical spaces are: (1) the usual $3 D$ point space (projective $3 D$ space); (2) the $4 D$ manifold of lines (represented in Plucker coordinates, or simple two-plane parametrization); the $3 D$ plane space (again projective $3 D$ space).

The transforms and inverse transforms from light density in one space to that in another are: the John (or X-ray) transform, $J$; the Radon transform, $R$; and a transform mapping from line space to plane space, denoted as $C_{23}$. In this paper we are constructing this last transform computationally.

\section{GENERATING THE RADON IMAGE}

The Radon image $\widehat{f}$ of a function $f$ in three dimensions is produced by integrating $f(x, y, z)$ over all possible planes [10,14]. As a result, the Radon image takes its value over the space of planes [9].

The equation of a plane in $3 D$ space $x, y, z$ is given by

$$
a x+b y+c z=r .
$$

where $(a, b, c)=\mathbf{n}$ is a unit vector normal to the plane. Therefore, the Radon transform in $3 D$ can be expressed as:

$$
\begin{gathered}
\widehat{f}_{a, b, c}(r)=\int_{-\infty}^{+\infty} \int_{-\infty}^{+\infty} \int_{-\infty}^{+\infty} f(x, y, z) \\
\times \delta(a x+b y+c z-r) d x d y d z .
\end{gathered}
$$

A parametrization of the plane, in terms of polar co-ordinates $r, \phi, \theta$ (see Fig. 3), is more useful for computing the inverse Radon transform. Expressing $(a, b, c)$ in terms of $(r, \phi, \theta)$ (see also Fig. 4), equation (2) becomes:

$$
\begin{aligned}
\widehat{f}_{\theta, \phi}(r) & =\int_{-\infty}^{+\infty} \int_{-\infty}^{+\infty} \int_{-\infty}^{+\infty} f(x, y, z) \delta(x \cos \phi \sin \theta \\
& +y \sin \phi \sin \theta+z \cos \theta-r) d x d y d z
\end{aligned}
$$

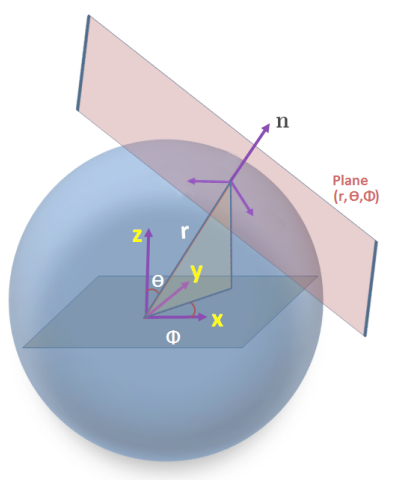

Fig. 3. Polar co-ordinate parametrization for plane

For applications to photography, a method is needed that would effectively integrate all rays that fall within the plane defined by the delta-function in (3). In this paper we will be describing a method to compute the Radon image from the light field captured with a traditional Plenoptic 2.0 camera [15]. Other devices may be used to capture the plenoptic function, including direct capture of the Radon image. Our current work focuses on theory and computation.

A simple model for the Plenoptic camera is described in Fig. 4 where the light rays after entering the camera through the main lens form a real image at plane P1. This image is re-imaged by each of the microlenses located at plane P2 - onto the sensor plane. We are interested in finding the sum over all rays that lie in an arbitrary plane $\mathrm{P}$, represented by (1) above.

To compute the integral of rays lying in plane $\mathrm{P}$, one needs to determine the corresponding pixels on the sensor plane where the rays are mapped by each of the microlenses. For this purpose, we determine the image of line $l_{1}$ as produced by arbitrary microlens, placed at $\left(x_{0}, y_{0}\right)$. The equation of line $l_{1}$ is given by:

$$
a x+b y=r .
$$




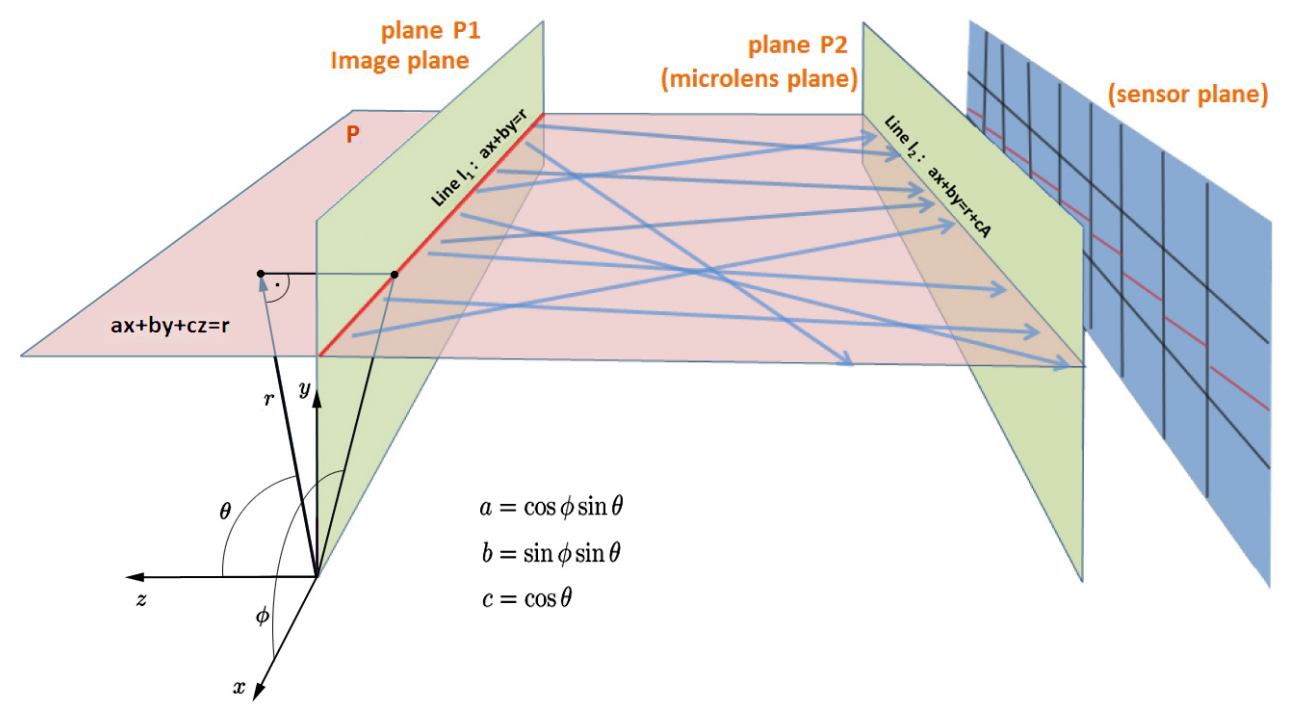

Fig. 4. Generating the Radon image from plenoptic camera data: Computing the transform from rays to planes involves summation over light rays lying in the plane of integration $P$ uniquely identified by lines 1 and 2 .

Similarly, the equation of line $l_{2}$ is given by:

$$
a x+b y=r+c A
$$

The image of a point $(x, y)$ by the microlens placed at $\left(x_{0}, y_{0}\right)$ is given by

$$
x_{1}=-M\left(x-x_{0}\right), \quad y_{1}=-M\left(y-y_{0}\right)
$$

where $\mathrm{M}$ is the magnification of the microlens. Substituting the values of $\mathrm{x}$ and $\mathrm{y}$ from the above equations into the equation of line $l_{1}$ written in the form $y=m x+d$, we get:

$$
y_{1}=m x_{1}-M\left(m x_{0}+d-y_{0}\right)
$$

Note that for this equation, the origin is fixed at the intersection of the optical axis of the microlens and the sensor plane, $M$ is the microlens magnification, and

$$
\begin{aligned}
& m=\frac{-a}{b}=-\cot \phi \\
& d=\frac{r}{b}=\frac{r}{\sin \phi \sin \theta} .
\end{aligned}
$$

The Radon image $\widehat{f}_{\theta, \phi}(r)$ is now obtained by summation over all pixels that lie on the line represented by (7), for each microlens intersected by plane P.

\section{THE INVERSE RADON TRANSFORM}

Given a function $f(x, y, z)$, the Radon image $\widehat{f}_{\theta, \phi}(r)$ is generated by integrating the function over all possible planes $(r, \phi, \theta)$. For selfconsistency of our paper, in this section we will derive the inverse Radon transform that takes the Radon image $\widehat{f}_{\theta, \phi}(r)$ as input and reproduces the function $f(x, y, z)$ as output (see [11]).

The $3 D$ inverse Fourier Transform equation can be written as:

$$
f(x, y, z)=\int_{-\infty}^{+\infty} \int_{-\infty}^{+\infty} \int_{-\infty}^{+\infty} F\left(\omega_{x}, \omega_{y}, \omega_{z}\right)
$$

$$
\times e^{2 \pi i\left(x \omega_{x}+y \omega_{y}+z \omega_{z}\right)} d \omega_{x} d \omega_{y} d \omega_{z} .
$$

With a change from Cartesian coordinates $\left(\omega_{x}, \omega_{y}, \omega_{z}\right)$ to polar co-ordinates $\left(\phi, \theta, \omega_{r}\right)$, where $\omega_{x}=\omega_{r} \cos \phi \sin \theta, \omega_{y}=$ $\omega_{r} \sin \phi \sin \theta$, and $\left.\omega_{z}=\omega_{r} \cos \theta\right)$, the equation becomes:

$$
\begin{gathered}
f(x, y, z)= \\
\int_{\phi=0}^{2 \pi} \int_{\theta=0}^{\pi} \int_{\omega_{r}=0}^{+\infty} F\left(\omega_{r} \cos \phi \sin \theta, \omega_{r} \sin \phi \sin \theta, \omega_{r} \cos \theta\right) \\
\times e^{2 \pi i \omega_{r}(x \cos \phi \sin \theta+y \sin \phi \sin \theta+z \cos \theta)} \omega_{r}^{2} \sin \theta d \omega_{r} d \theta d \phi .
\end{gathered}
$$

However, by the Fourier Slice theorem (see [11], pp. 163)

$$
F\left(\omega_{r} \cos \phi \sin \theta, \omega_{r} \sin \phi \sin \theta, \omega_{r} \cos \theta\right)=\widehat{F}_{\theta, \phi}\left(\omega_{r}\right)
$$

where $\widehat{F}_{\theta, \phi}\left(\omega_{r}\right)$ is the Fourier transform of $\widehat{f}_{\theta, \phi}(r)$, eq. (3).

Substituting (9) in (8),

$$
\begin{gathered}
f(x, y, z)=\int_{\phi=0}^{2 \pi} \int_{\theta=0}^{\pi} \int_{\omega_{r}=0}^{+\infty} \omega_{r}^{2} \widehat{F}_{\theta, \phi}\left(\omega_{r}\right) \\
e^{2 \pi i \omega_{r} r} d \omega_{r} \sin \theta d \theta d \phi
\end{gathered}
$$

where $\mathrm{r}$ is given by $r=x \cos \phi \sin \theta+y \sin \phi \sin \theta+z \cos \theta$

However, since

$$
\int_{\omega_{r}=0}^{+\infty} \omega_{r}^{2} \widehat{F}_{\theta, \phi}\left(\omega_{r}\right) e^{2 \pi i \omega_{r} r} d \omega_{r}=\frac{-1}{4 \pi^{2}} \frac{\partial^{2} \widehat{f}_{\theta, \phi}(r)}{\partial r^{2}}
$$

the expression determining the inverse Radon Transform becomes:

$$
f(x, y, z)=\frac{-1}{8 \pi^{2}} \int_{\phi=0}^{2 \pi} \int_{\theta=0}^{\pi} \frac{\partial^{2} \widehat{f}_{\theta, \phi}(r)}{\partial r^{2}} \sin \theta d \theta d \phi
$$

It can be easily shown [16] that (10) is equivalent to:

$$
f(x, y, z)=\frac{-1}{8 \pi^{2}} \nabla_{x, y, z}^{2}\left(\int_{\phi=0}^{2 \pi} \int_{\theta=0}^{\pi} \widehat{f}_{\theta, \phi}(r) \sin \theta d \theta d \phi\right)
$$


Notice that this expression has the same form as the Poisson equation in electrostatics, with $f$ playing the role of charge density:

$$
f=-\nabla^{2} f^{1} .
$$

Here, the backprojected Radon image $f^{1}$ is defined as

$$
f^{1}(x, y, z)=\frac{1}{8 \pi^{2}} \int_{\phi=0}^{2 \pi} \int_{\theta=0}^{\pi} \widehat{f}_{\theta, \phi}(r) \sin \theta d \theta d \phi,
$$

and takes the role of the electric potential.

\section{ALGORITHM}

The major steps of our algorithm are: 1) Pe-compute the Radon image as described in Section 3; 2) Compute the back projection $f^{1}(x, y, z)$ as described in Section 4; and 3) Compute the density of virtual sources $f(x, y, z)$ by applying the Laplacian of Gaussian operator described in equation (12) followed by denoising. The final step is needed because the result after backprojection $f^{1}(x, y, z)$ is a blurred version of the function of interest $f(x, y, z)[16,17]$.

The range of values used for various parameters are:

1. $\phi$ : We vary $\phi$ in steps of $1^{\circ}$ from $0^{\circ}$ to $360^{\circ}$. This sampling is sufficient for the images we captured using a Plenotic camera, which have a microimage size of $75 \times 75$.

2. $\boldsymbol{\theta}$ : Variation in $\theta$ contributes to depth $z$. Considering the small aperture size, $\theta$ varies from $80^{\circ}$ to $100^{\circ}$ with a step of $1^{\circ}$. If $\theta$ was fixed to $90^{\circ}$, by varying only $\phi$ and $r$ during back projection, we would get only depth-independent reconstruction: $r=x \cos \phi \sin \theta+y \sin \phi \sin \theta+z \cos \theta$ would become a function of $(x, y)$ only.

3. $\mathbf{r}$ : We vary $r$ in steps of size one. By the Nyquist criterion, the maximum value $r$ can have $\left(r_{\max }\right)$ is two times the pixel size of the final image.

\section{RESULTS}
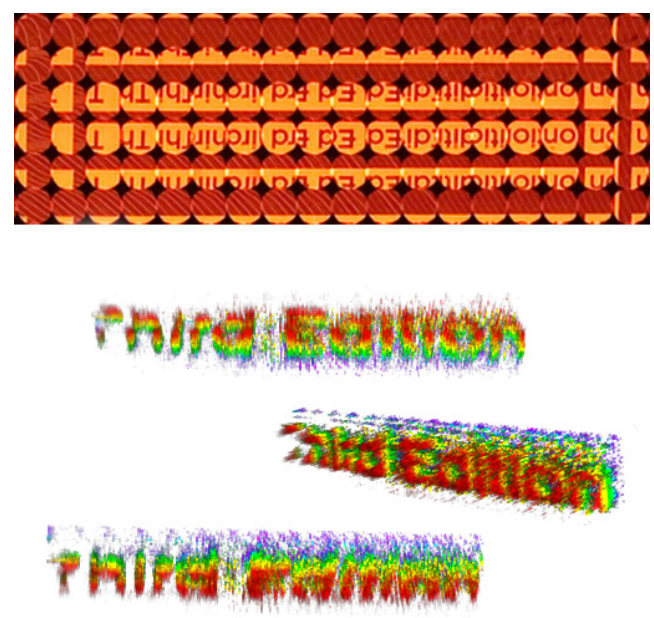

Fig. 5. Point cloud with full 3D information recovered from a Radon plenoptic image using formula (11). Plenoptic camera data that has been used to generate the Radon image is shown on top. Three views of generated point cloud are shown below, color representing depth.

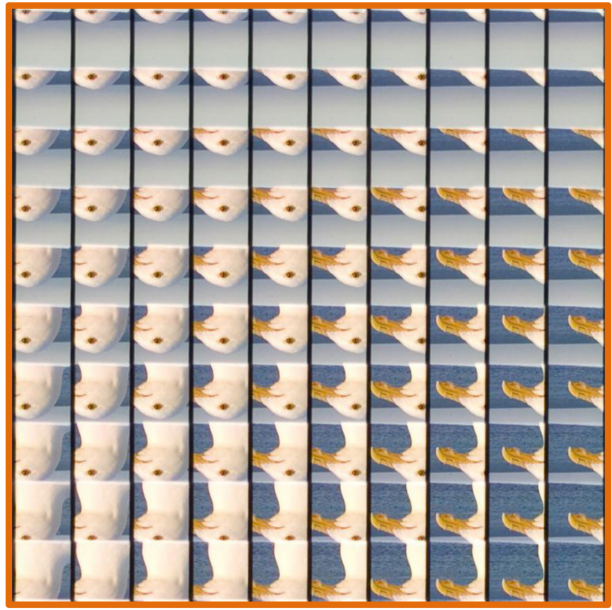

(a)

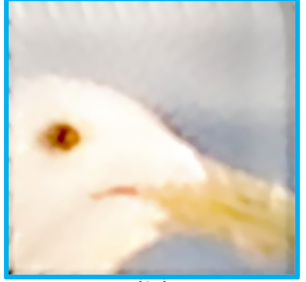

(b)

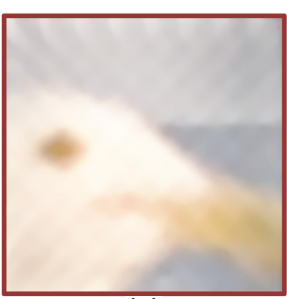

(c)
Fig. 6. (a) Input plenoptic camera data, and the backprojected image at two different depth planes, (b) seagull and (c) ocean. The input plenoptic image was captured with a focused plenoptic camera [15], so individual microimages are inverted.

Plenoptic cameras have attracted significant recent attention because of the ability to, in essence, frame pictures computationally (rather than optically) and adjust parameters such as parallax and focus after the fact [18]. Our preliminary results demonstrate that such effects are possible using the Radon plenoptic function. Figure 5 shows a point cloud with full 3D information recovered from a Radon plenoptic image using formula (11). The Radon image is generated from data captured with a traditional plenoptic camera. Figure 6 shows the standard "Seagull" plenoptic image rendered by performing filtered backprojection $[11,12,16]$ at the foreground plane and at the background plane.

\section{CONCLUSIONS}

This paper introduces the Radon plenoptic function (RPF) as a new representation for the plenoptic function over plane space instead of the traditional representations over point space or line space. The $\mathrm{RPF}$ is related to energy density (over point space) and the light field (over line space) by the inverse Radon transform and the X-ray transform, respectively. Although the new representation is lowerdimensional (and therefore more compact) than the traditional lightfield representation, traditional rendering effects such as parallax and variable focus are still achievable. The experimental results presented here demonstrate the potential of this approach. Future work will further develop the theoretical and computational foundations for the RPF that were presented here. 


\section{REFERENCES}

[1] E. Adelson and J. Bergen, "The plenoptic function and the elements of early vision," in Computational Models of Visual Processing. 1991, MIT Press.

[2] Todor Georgiev, Ke Colin Zheng, Brian Curless, David Salesin, Shree Nayar, and Chintan Intwala, "Spatio-angular resolution tradeoffs in integral photography.," Rendering Techniques, vol. 2006, pp. 263-272, 2006.

[3] Marc Levoy and Pat Hanrahan, "Light field rendering," ACM Transactions on Graphics, pp. 31-42, 1996.

[4] G Lippmann, "Epreuves reversibles donnant la sensation du relief," Journal of Physics, vol. 7, no. 4, pp. 821-825, 1908.

[5] Anat Levin and Fredo Durand, "Linear view synthesis using a dimensionality gap light field prior," in Computer Vision and Pattern Recognition (CVPR), 2010 IEEE Conference on. IEEE, 2010, pp. 1831-1838.

[6] Ren Ng, Digital Light Field Photography, Ph.D. thesis, Stanford University, 2006.

[7] José G Marichal-Hernández, Jonas P Lüke, Fernando L Rosa, and José M Rodríguez-Ramos, "Fast approximate 4d: 3d discrete radon transform, from light field to focal stack with o (n4) sums," in IS\&T/SPIE Electronic Imaging. International Society for Optics and Photonics, 2011, pp. 78710G-78710G.

[8] Fritz John, "The ultrahyperbolic differential equation with four independent variables," Duke Mathematical Journal, vol. 4, no. 2, pp. 300-322, 061938.

[9] Johann Radon, "Über die bestimmung von funktionen durch ihre integralwerte längs gewisser mannigfaltigkeiten,"
Berichte über die Verhandlungen der Königlich-Sächsischen Akademie der Wissenschaften zu Leipzig, MathematischPhysische Klasse, pp. 262-277, 1917.

[10] A. Macovski, Medical Imaging Systems, Prentice-Hall, Inc., Upper Saddle River, NJ 07458, 1983.

[11] Thorsten M. Buzug, "Computed tomography," in Springer Handbook of Medical Technology, pp. 311-342. Springer, 2012.

[12] Sigurdur Helgason, Radon Transform, Birkhauser, 1999.

[13] A Gershun, "Light field," Translated by P. Moon and G. Timoshenko in Journal of Mathematics and Physics, vol. 18, pp. 51-151, 1936.

[14] William L. Briggs and Van Emden Henson, The DFT, An Owner's Manual for the Discrete Fourier Transform, SIAM, 1995.

[15] Andrew Lumsdaine and Todor Georgiev, "The focused plenoptic camera," in IEEE International Conference on Computational Photography (ICCP). IEEE, 2009, pp. 1-8.

[16] Stanley R. Deans, The Radon Transform and Some of its Applications, Dower, 1983.

[17] T. M. Buzug, Computed Tomography, Springer, 2012.

[18] R. Ng, M. Levoy, M. Brédif, G. Duval, M. Horowitz, and P. Hanrahan, "Light field photography with a hand-held plenoptic camera," Tech. Rep. CSTR 2005-02, Stanford Univ, Apr. 2005. 